\title{
A PROPOSITO DI UNA QUESTIONE SULLE ONDE MICROBARICHE CARATTERISTICHE DEI FRONTI
}

\author{
F. MoserTI
}

Velle svariate ricerche sulle onde microlariche che da qualche anno stiamo conducendo, abliamo fatto via via uso di strumenti sempre più perfezionati. Dapprima ablyiamo adoperato il rudimentale, sia pure ingregrnoso, strumento ideato da Padre Allani, più tardi quello del Bossolasco, entrambi a registrazione su carta affumicata; infine strumenti a registrazione fotografica da noi stessi costruiti. Albliamo ben presto cessato di usare il microbarografo Alfani e fummo indotti a riò dopo aver visto che questo strumento era utile soltanto alla registrazione di brevi oscillazioni della pressione (fino a qualche minuto di feriodol dipendenti massimamente dal vento. Questo strumento puo renir usato con profitto come turlografo, per lo studio della turloolenza atmosferica in relazione a varie situazioni meteorologiche ma mal si presta alla registrazione delle fluttuazioni bariche a lungo periodo. Le onde più lunghe (di periodo dell'ordine di alcune decine di minutil, che sono di gran lunga le più interessanti, o non vengrono registrate sufficientemente ampie dall'Alfani, causa la sua costante valvolare elevata l'amplificazione, per $i$ microbarografi lis ha un andametito proporzionale a $\omega / \omega^{2}+A^{2}$, più elevato è $A$ più piccolo. a parita di periodo, risulta l'ingrandimentol, o la loro registrazione non è comoda da seguire causa il troppo veloce scorrimento del regristratore. Sui nostri fotomicrobarografi è molto più facile e comodo rilevare le onde lunghe e si può chiaramente notare la loro forma e il loro comportamento. Cosi abljiamo visto $(\bar{\prime} /$ che, ogni qualvolta passava sulla stazione, o nelle vicinanze di essa, un fronte, fosse esso caldo, freddo od occluso, si notava la comparsa di ondulazioni di periodo tra 15 min e $l$ h circa, irregolari e frastagliate, di solito molto ampie, che si sovrapponevano al sulsstrato oscillante 'normale' doruto ad altri fattori. Tutte le volte, cioè, che si nota, sul bollettino sinottico dello stato del tempo $t^{t}$ i il passaggio di un fronte su una data stazione fo tutte le volte che, da questo loollettino, il tempo di passagrio della linea frontale può esser dedotio in lase alle sue 
posizioni precedenti e seguenti rispetto alla stazionel, si trovano al microharografo le caratteristiche onde frontali a lungo periodo. Ia figura l.t è un chiaro esempio di alcune di queste oscillazioni scelte a caso dalla nostra ricea raccolta. Si vede chiaramente specie nelle registrazioni dei giorni $28-29$ settembre 1951 e 6-7 marzo 1952 come si passa, in corrispondenza al transito di una lines frontale I figr $I B$, dal liscio e regolamente ondulato microbarogramma "nor-

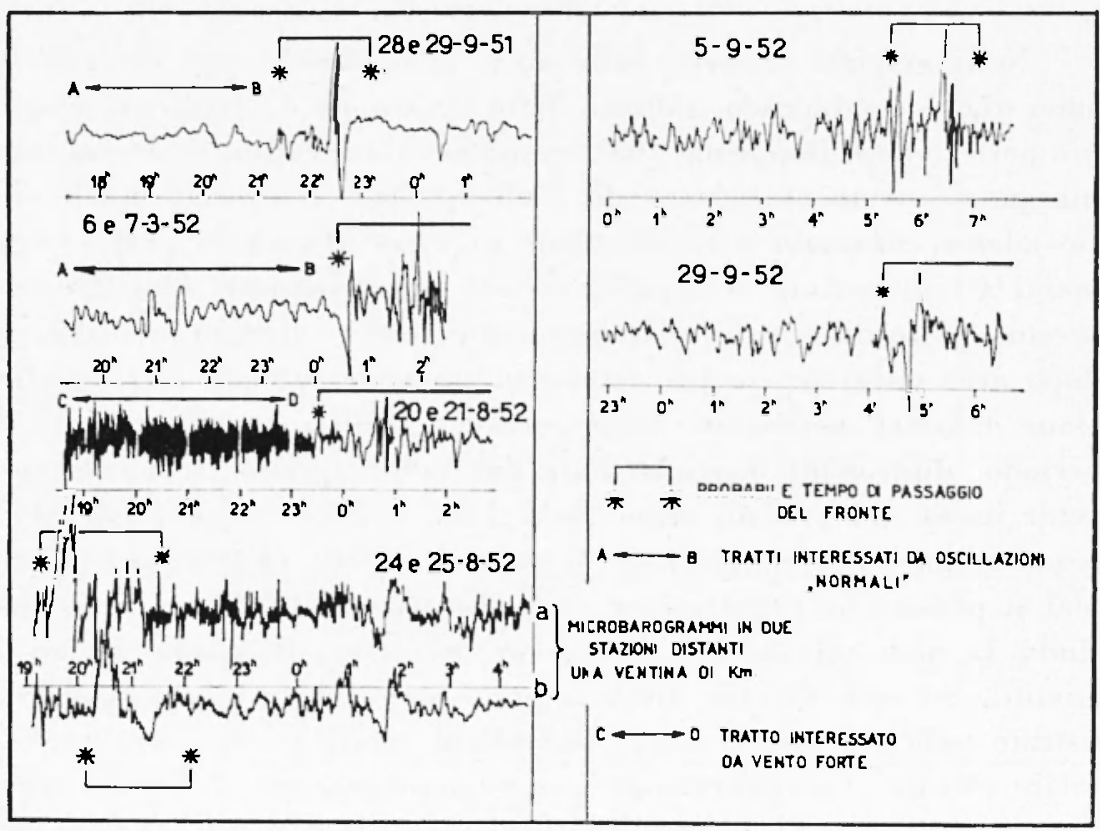

Fig. 1il

male', al microbarogramma 'perturbato'. Invertendo le premesse di quanto abbiamo detto possiamo asserire che: ogni qualvolta sulla trarcia microbarografica si riscontra il gruppo (o $i$ gruppi) donde caratteristico del fronte si ha effettivamente il passuggio di una tale discontinuita sulla stazione. Reti di stazioni microharografiche annesse alle normali stazioni meteorologiche potrebloro cosi rilevare, molto comodamente, il passaggio della linea frontale te, speso, anche pre-

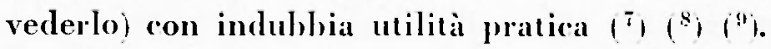

Molto spesso, se pur non sempre, il passaggio di un fronte $\dot{c}$ accompagnato dallo scatenersi di violenti temporali, altre volte, invece, notiamo al microharografo onde da fronte anche con tempo non ec- 
crssivamente brutto tanto che, se non trovassimo conferma sui bollettini sinottici, dubiteremmo quasi noi stessi dell'avvenuto passagrio. Il temporale di per se stesso, anche se non è collegato ad un fronte, frovoca turbamenti microbariei a breve e lorevissimo periodo (qualrhe secondo e menof e anche a forte ampiezza, che ne rappresentano la caratteristica principale $(7)$; tali registrazioni si ottengono col mi-
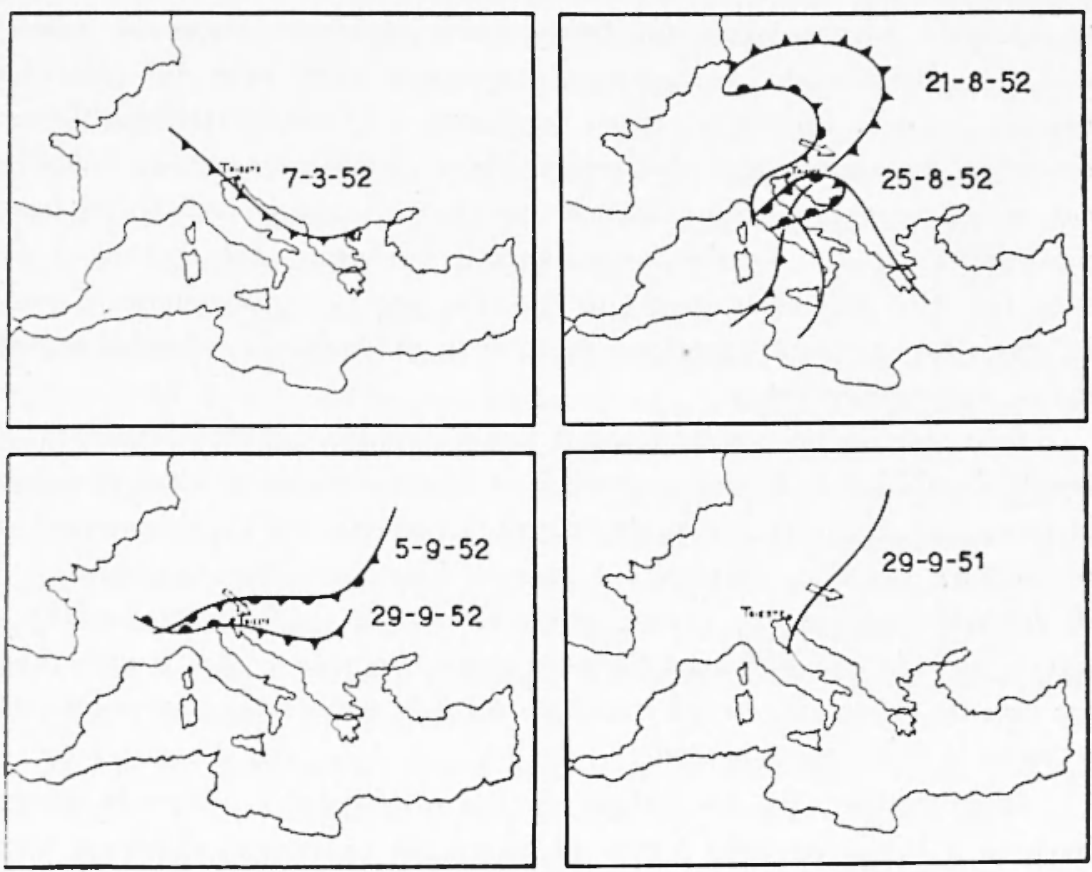

Fig. $1 b$

croharografo dlfani che i lo strumento ideale per la percezione di onde brevi. Quando il temporale è legato al passaggio di un fronte, le onde lunghe caratteristiche di questo passaggio si manifestano anche sullo strumento Allani, benché non chiaramente, perché molto ridotte d'ampiezza e più o meno mascherate dalle oscillazioni a breve periodo. Queste onde lunghe, del resto, si notano anche sui normali barowrafi. Abliamo infatti $\left({ }^{10}\right)$ scomposto in cicli semplici (previo ingrandimentol alcuni harogrammi ottenuti durante temporali e al). hiamo visto, mescolati e mascherati fra le altre periodiciti, alcuni sruppi di onde fortemente esaltate in ampiezza che presentano i me- 
desimi periodi osservati direttamente al microbarografo nella medesima circostanza.

Segno, dunque, caratteristico, del passagrgio di un temporale i la registrazione mierohariea di particolari onde od impulsi di hrevissimo periodo e forte ampiezza. Tali onde derivano dal vento più o meno impetuoso, soffiante quasi senza direzione privilegiata durante il temporale, e si notano anche senza il contemporanco passagrio del fronte. Quando invece passa un fronte sulla stazione, anche se questo non i accompagnato da temporali, vengono registrate dal microbarografo le onde lunghe a gruppi irregolari e frastagliati che abbiamo descritto. Le onde brevi del temporale si mettono bene in evidenza col microbarografo Allani, adatto appunto alla registrazione di hrevi periodiciti; quelle lunghe, segnalanti il passaggio del fronte, si notano con altri microbarografi più specifici per la registrazione di onde lunghe. Due strumenti mettono dunque in evidenza due diversi aspetti dello stato atmosferico.

Che particolari onde lunghe siano legate significativamente ai fronti, lo abbiamo del resto messo in evidenza anche in altra maniera. Il passaggio di fronti sul mare ingenera, infatti, $($ ) $)(*)$ mierosismi di

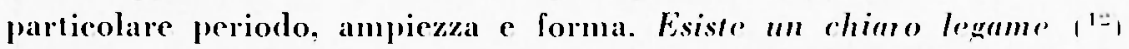
di inferdipendenza tra questi microsismi " lo onde microbariche a lango periodo che abbiamo definito come dipendenti dai fronti, mentre non ne esiste alcuno tra i microsismi ole oscillazioni barometrich" " breve o brevissimo periodo.

In proposito alla descrizione da noi effettuata $\left.\uparrow^{\prime}\right)$ di onde mierobariche a lungo periodo legate al passagrio di fronti, abbiamo letto un lavoro (10) dello Zanon, ove l'Autore si dice non in accordo coi nostri concetti. Sulle nostre asserzioni non abbiamo da ricrederci. Lo Zanon piuttosto, in aleuni lavori precedenti $\left.(1:), 1^{1-1}\right)$ in eui trattava dell'applicazione pratica dei microbarografi, pur usando gli strumenti Alfani lassai poco sensibili, come si disse, per le onde lunghei. era riuscito ad intravedere, $c$ descrisse pure lui, un legame fra queste oscillazioni atmosferiche a lungo periodo e il passagrio di fronti 10. più genericamente, con la presenza di temporali c di zone perturbater. Appare comunque chiaro da questi primi lavori dello Zanon l’importanza che l'Autore da alle onde di lungo periodo e la natura contingente c affatto caratteristica giustamente attribuita alle brevi oscillazioni. In un suo ultimo lavoro $1 \%$, invece, pur trattando in parte proprio sugli stessi casi gia precedentemente descritti, THutore di- 
mentica quasi lesistenza delle interessantissime onde lunghe e si pone a voler dimostrare la presenza di relazioni tra onde microhariche a brevissimo periodo e il passaggio di un fronte, e cio per confermare analoghe asserzioni di due Autori americani $\left({ }^{1},{ }^{11}\right.$, che ricercarono con microharografi elettromagnetici del tipo di quello costruito dal Macelwanc: 3 . Queste correlazioni tra onde brevi e passagrio del fronte sono, a nostro aviso, del tutto illusorie. Esse non dipendono da una caratteristica propria della discontinuita ma solo dal vento che. con maggiore o minore intensita, l'accompagna. Gli strumenti elettromagnetici usati dagli americani sono, ancor più strettamente del microbarografo Alfani, legati alla sola registrazione di onde brevi e necessariamente quindi, i predetti Autori non poterono individuare il legame esistente tra il passaggio del fronte e i vistosi gruppi di impulsi microharici con periodo tra $15 \mathrm{~min}$ e $l h$ circa che ne sono la caratteristica essenziale. Essi si spinsero quindi in poco significative ricerche sulle hrevi oscillazioni da vento che a volte accompannano il passaggio della discontinuità ma non rappresentano affatto una caratteristica degna di nota. Noi siamo certi ormai, dopo aver controllato ugulche centinaio di casi, delle nostre asserzioni esposte in l') e qua megrlio definite. Anzi, insistiamo che venga applicato con finalita pratirhe (di indubbia importanza) lo studio e linterpretazione di queste oscillazioni a lungo periodo cosi significativamente legate al passaggio della linea frontale.

In fig. 1A riportiamo alcuni casi di onde microbariche da fronte e in figura 1B, per confronto. le posizioni dei fronti relative alla stazione di Trieste alle ore 1 del griorno segnato $1 \%$. Confrontando con queste posizioni il tempo di registrazione degli impulsi microharici qua descritti, o estrapolando da queste posizioni il probahile tempo di passagrgio del fronte, si può osservare senza ombra di crrore la perfetla concordanza con le notre precedenti asserzioni.

Istituto Vaz. di Geofisica - Osserv. Geofisico di Trieste - Maggio 39.54.

\section{RI.ASSL NTO}

Poiche alcuni autori. usando apparecchiature poco idones allo scopo. credettero di trovare un certo legame fra le onde microbariche " breve periodo "il passaggio di fronti sulla stazione registratrice, 
abbiamo qua insistito sul fatto che laspetto microburografico caratte. ristico del passaggio di an fronte ì rappresentato da particolari onde " lungo periodo.

\section{$S U M M A R Y$}

Since some authors, using instruments few idoneous to the aim, believed to find a certain connexion between microbaric waves of short periode and the transit of fronts over the registering station, we have hier insisted on the fact that the microburographic aspect of the passage of a front is represented from particulares waves of long periode.

\section{BIBLIOGRAFIA}

(1) CaAlk R. D. M., Atmospheric microoscilations. Journ. Meteor. $i$ (1950).

(-) D’Henry G. e Moret.1 C.., Sulle muse dei microsismi. Ann. Geof., II, 2 (1949).

(:) Mackewax: J. B. e Ranut:z E., The electromagnetic microbarograph and its performance. Trans. Amer. Geoph. Union. 19 (1938).

(t) Ministero dell'Aeronatutia, Bollentino quotidiano dinformazioni del Servizio Meteorologico dell teronautica. Roma 1951.52.

(i) Monelat C., Contributo allo studio dei microsismi. Ann. Geof. I. + (1918).

(fi) Mosetti F., Teoria del microbarograjo AlJuni. Ann. Geof. IV, I (1951).

(i) Mosetri F., Le onde microbariche. Riv. Meteor., Aeron. 12, III 11952).

(n) Mosetri F., Primi risultati sulla localizzazione delle perturbazioni atmosseriche in base allo studio delle onde microbariche. Ann. Geof., VI. + 11953$).$

(9) Mosetti F. Uheriori elementi per la localizazione delle perturbazioni amosferiche in base alla direzione di provenienza delle onde microbariche. Riv. Meteor. Aeron., XIV, 2 1954.

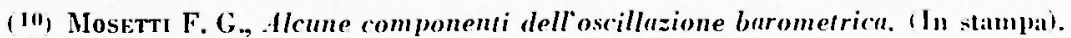

(11) Roscitke H. The relation between fir-pressure microoscillation and con. current Synoptic Pafterns. Jour. Meteor. 9 (1952).

(1-) Vencelci F. e Mosettr F., Osservazioni sui rapporti tra onde microbariche. onde microburografiche e microsismi. Arad. Pout. Scient. Semaine d’ótude sur le problème des microseismes, nov. 1951.

(1.3) Zavos F. S., Pulsazioni della pressione amosferica " periodo brevissimo. Ann. Geof. II, 1 (1919).

(1t) Zanon F. S., Fronti nerologici e stato biologico. Areht. Osped. al Mare. IV, 1950 .

(1ii) Zavos F. S., $1 /$ microbarografo Ilfani ed $i$ iromi aerologici. Geof. pura e appl., 27 (195.). 